Shuo Li', Cheng Yao', Mingxuan He', Qingcong Wang', Ying Wang', Yuyu Lin', Juanli Liu',

Fan Xia ${ }^{2}$, Leijing Zhou'

1Zhejiang University, Hangzhou, China, ${ }^{2}$ Mercyhurst Preparatory School, Erie, PA, USA

leesoo_shuo@outlook.com, \{Yaoch, 21851224, 21851221, linyuyu, liulijuan\}@zju.edu.cn,

jerryxia26@163.com,leijing @zju.edu.cn

\title{
Swimming Coach: An Immersive Swimming Learning System
}

\begin{abstract}
Swimming is a common activity to keep fit. To assist hydrophobic users to overcome the fear of learning swimming, and to give full play to the advantages of land swimming practice, we propose a swim learning system which includes VR system, wearable devices and frame structure to provide users with an immersive and interactive learning experience of swimming without water. Users' real-time postures can be detected by using the body gesture recognition system, while visual and haptic feedback are also available to users. Preliminary investigation shows that the proposed system has demonstrated potential in the field of swimming learning.
\end{abstract}

\section{Keywords}

Swimming Learning, Virtual Reality, Immersion, Head Mounted Display, Gesture Recognition

\section{Introduction}

Swimming is a popular activity that offers physical and mental benefits. It ranks as one of the sports activities that people like most [1, 2]. People learn to swim not only for health and recreation [3] but also for selfrescue [4]. However, swimming is a complex sport that requires a flow of quick, smooth and accurate movements, so swimmers need external guidances and constant practices to build muscle memory [5] by land swimming practice. Due to the lack of related scientific learning system, beginners are hard to overcome the fear of water. Besides, they do not receive immersive swimming feedback, which do not suffice for fast improvements.

Thus, we propose "Swimming Coach," an immersive swimming learning system. The system can help beginners to learn to swim with an immersive and interactive swimming learning experience.

\section{Related Work}

Some researchers created virtual immersive underwater environments to mimic the real visual feeling. For instance, Shogo Yamashita et al. designed a cave system with multiple stereoscopic projections on surrounding acrylic walls, providing swimming environment with swappable scenes, such as coral reefs and shark cage diving [6]. Similarly, Lisa Blum et al. presented a system using augmented reality techniques to simulate a regular swimming pool with virtual objects [7]. The above works made users feel that they were in water environments visually, though researchers did not provide other sensory experiences.

Some works used certain physical structures to simulate 
swimming in water under the condition of waterless. In the system of Tzu-Pei Grace Chen et al., the user was suspended in a real swimming apparatus which simulated the swimming condition in a virtual Pacific Ocean environment [8]. Dhruv Jain et al. also used the suspended system, but they placed an inflatable airbag under the user's belly, which allowed the user's body to rise and fall in sync with breathing [9]. These two works allow users to move limbs, but they do not include swimming poses in different scenes.

To create a real experience for users, and to let users realize the transition from not being able to swim to swimming, the feeling of immersion and presence of users should be taken into account when designing the swimming learning system [10].

\section{System Design}

Based on previous works, we designed "Swimming Coach", which includes four components: body support prototype, Head Mounted Display, multi-scene swim learning application, as well as human gesture recognition and matching module. Our system can help swimmers to learn swim in various swimming scenarios, and learn multiple postures (competitive swimming and practical swimming).

\subsection{Swimming Support Prototype}

We designed and made a body support prototype. In this support prototype, a support frame is attached to the user by a harness which allows the user's hands and feet to move freely. Notably the support frame can be rotated so that the user can learn how to swim in any vertical angles.

To simulate water resistance, we designed a wristband (Fig. 1), which is connected to the safety belt through an elastic band. What's more, to simulate the feeling of floating in the water, we added an inflatable air cushion on the support prototype (Fig. 2). The inflatable air cushion is connected to an inlet air pump and an outlet vacuum pump through the air pipe. When users are learning, they can experience the feeling of flotation through the expansion and contraction of the air cushion.

\section{2 (Head Mounted Display) HMD}

To enhance the feeling of immersion and increase the interaction of "Swimming Coach" provided by tactual, visual and auditory feedback, we develop the HMD (Fig.1). It includes VR glasses and a breathing mask with an airbag.

We set the completion time for each set of actions,
Fig. 1. A user is wearing HMD and experiencing Multi-scene swimming learning.

Fig. 2. The inflatable air cushion.
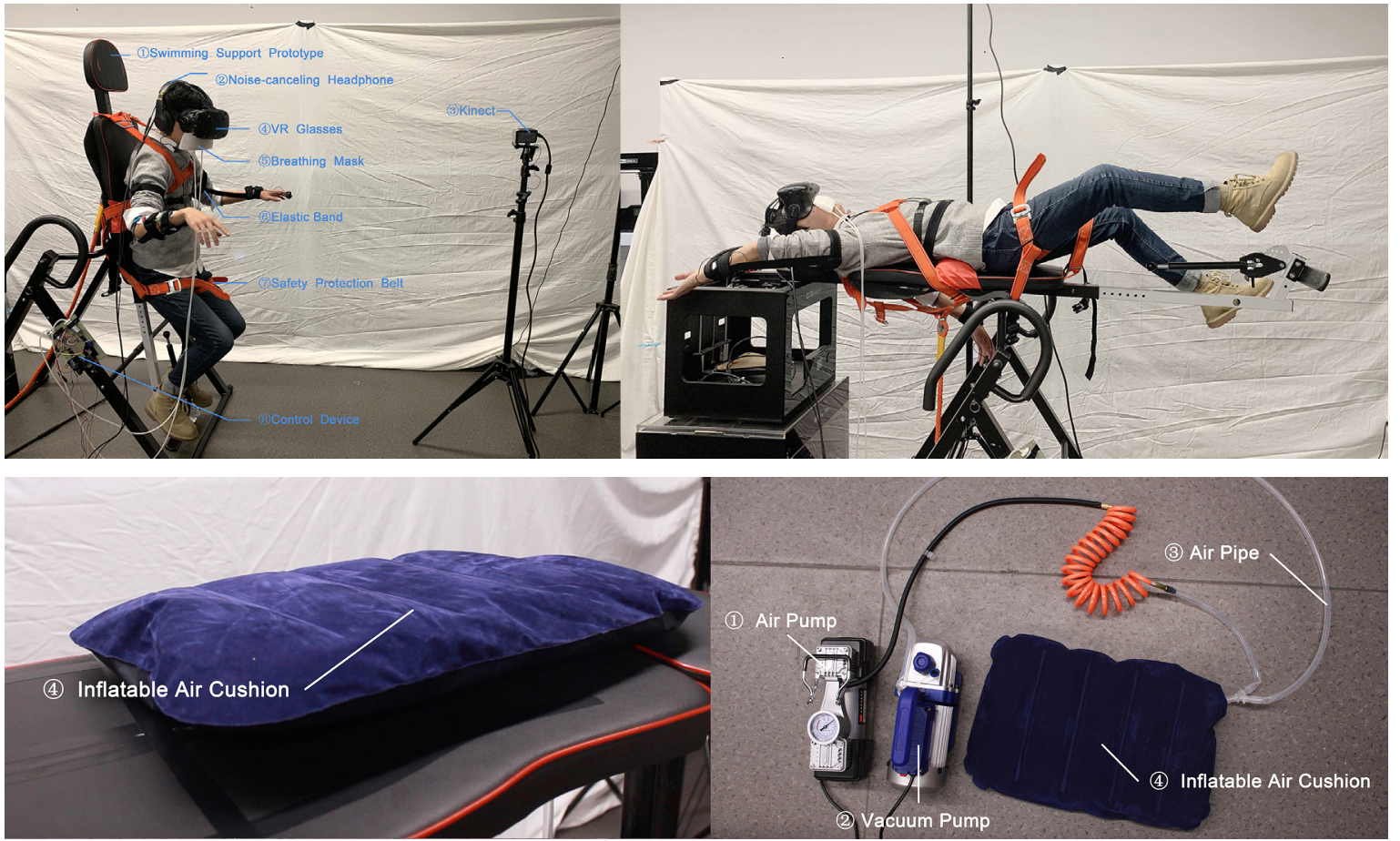
during which the user needs to complete the corresponding action.

In the VR glasses, the user's main field of view is his or her first-person view (Fig.3). The upper left corner of the interface is the matching between the image from the third-party perspective and the correct posture. If the user's action is inconsistent with the standard action, the interface will present voice prompt.

The airbag of the breathing mask (Fig.4) is prompting the user to inhale and exhale correctly while swimming. The time interval between airbag expansion and contraction is consistent with the standard swimming breathing rate. We use this device to prompt the user to inhale and exhale correctly while swimming.

\subsection{Human Gesture Recognition and Matching Module}

Based on Kinect and Unity, we designed a set of human gesture recognition and matching module, which can identify the user's bone nodes in real time, and then match the recognition results with the bone nodes of standard actions to determine whether the user's swimming action is correct or not. VR glasses will also give users corresponding hints.

\subsection{Multi-scene Swimming Learning Application}

According to previous research, locations of drowning by different age groups were categorized into the swimming pool and open water (such as a lake, river, or pond) [11]. In our system, we use multiple scenes in VR vision to help users practice swimming in different kinds of water. We use Unity to create our scenarios, such as the swimming pool and ocean (Fig. 5). Besides, we design an oxygen value (green bar) and a health value (blue bar). This setting can simulate the real status of swimmers in the water and provide data for the HMD.

\section{Evaluation}

20 volunteers (female 7 , male 13) with an average height of $173 \mathrm{~cm}(S D=9.2)$ and an average weight of $58 \mathrm{~kg}(S D=11.7)$ were recruited from Zhejiang University, China. In order to test the effectiveness of the system. (e.g. In the experiment, the volunteers used the Swimming Coach one by one for 30 minutes

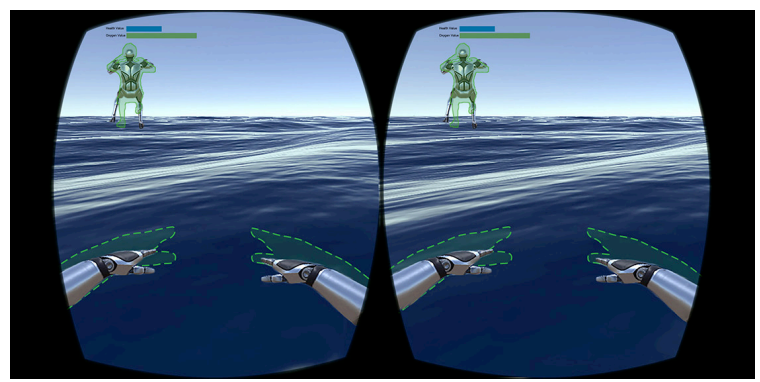

Fig. 3. A scene from the HMD when users learn freestyle swimming.

under proper instructions from the researchers. The volunteers tested two different swimming scenarios). After experiencing this system, the volunteers were asked to complete a questionnaire [12] and then interviewed concerning their feelings and feedbacks about the swim learning system. The data were later analyzed using a chi-square $(X 2)$ test [13]. The reported overall rating of presence across all participants was $4.79 / 7$ ( $S D=1.59)$. It indicated that they were positive about the system and felt immersed.

Meanwhile, according to the interview, 17 of 20 volunteers can learn the basic swimming posture, 3 volunteers said the discomfort caused by the safety belt could affect learning to swim.

\section{Conclusion and Future Work}

In this paper, we present an immersive and interactive VR swim learning system, a combination of HMD, human gesture recognition system and multiscene swimming learning application. Through the experiment of 20 participants, the system proves to be effective to swimming learners. However, we are aware that certain problems do exist. For example,

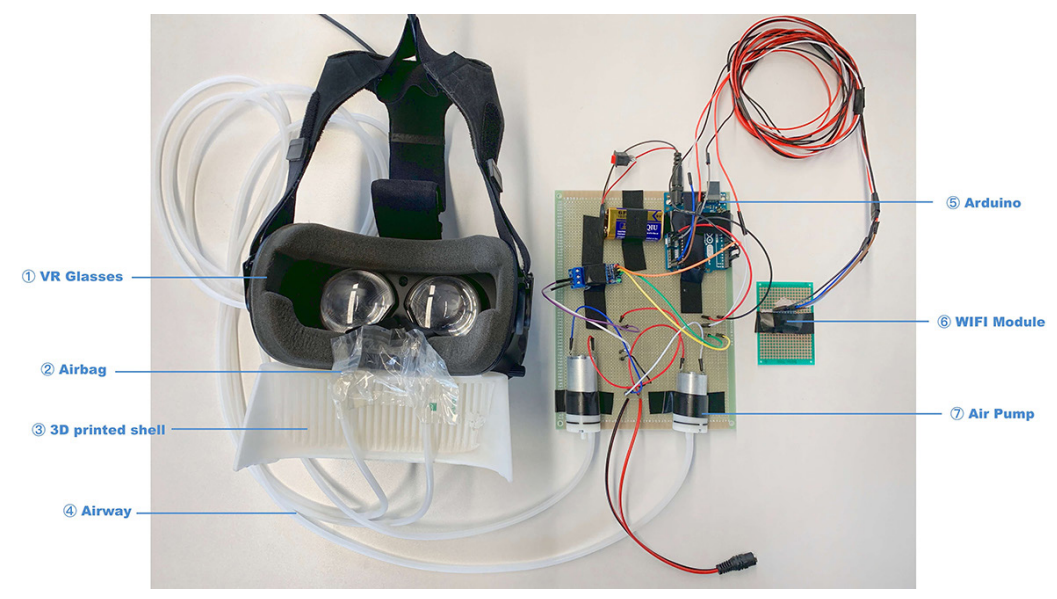

Fig. 4. The breathing mask. 
Fig. 5. Multi-scene: swimming pool and ocean

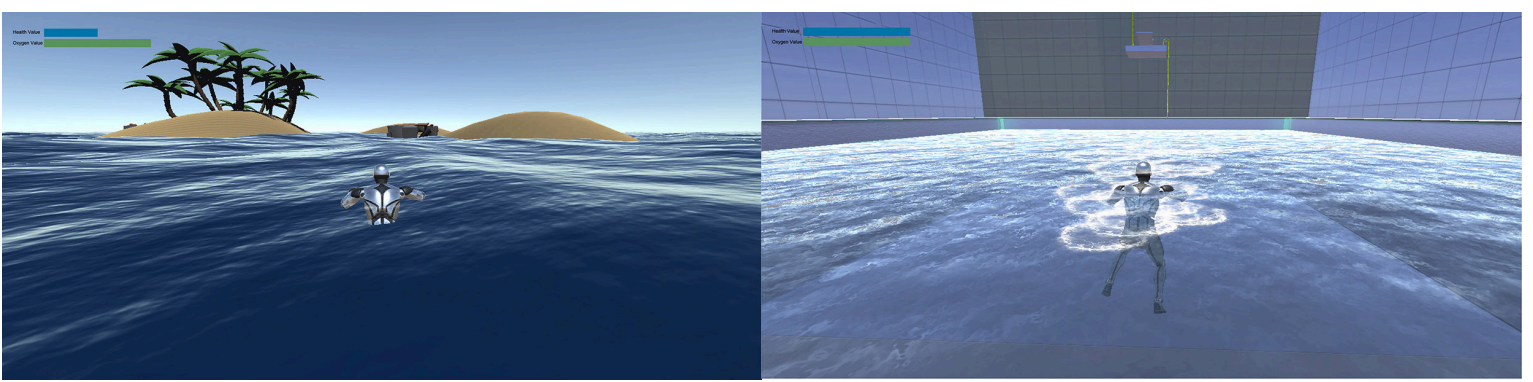

the swimming movements of participants often made wrong movements, leading to the massive warning voice prompt from the system, which might impair the immersion experience of the users.

In future work, we will improve the body support prototype, and change the form of error reporting from voice prompts to color changes of characters in VR scenario, and conduct more tests to perfect our "Swimming Coach". Besides, we plan to upgrade this equipment into a more general device, which can be used for parachuting, gliding and other sports.

Acknowledgements. The authors thanks all the reviewers for providing valuable insights and suggestions that have helped in substantially improving this paper, as well as all volunteers for general support. This project is supported by the National Natural Science Foundation of China (Grant No. 61332017).

\section{References}

1. da Costa, L. P., \& Miragaya, A. (Eds.). (2002). Worldwide experiences and trends in a sport for all. Meyer \& Meyer Verlag.

2. Helen Jones, Peter Millward, Babtunde Buraimo. (August 2011). Adult participation in sport. Department for Culture, Media and Sport.

3. Katz, J. (2003). Your water workout.

4. Szpilman, D., Bierens, J. J., Handley, A. J., \& Orlowski, J. P. (2012). Drowning. New England journal of medicine, 366(22), 2102-2110.

5. Krakauer, J. W., \& Shadmehr, R. (2006). Consolidation of motor memory. Trends in neurosciences, 29(1), 58-64.

6. Yamashita, S., Zhang, X., \& Rekimoto, J. (2016, October) Aquacave: Augmented swimming environment with immersive surround-screen virtual reality. In Proceedings of the 29th Annual Symposium on User Interface Software and Technology (pp. 183-184). ACM.

7. Blum, L., Broll, W., \& M $\square$ ller, S. (2009, August). Augmented reality under water. In SIGGRAPH'09: Posters (p. 97). ACM.

8. Chen, T. P. G., Kinoshita, Y., Takama, Y., Fels, S., Funahashi, K., \& Gadd, A. (2004, August). Swimming across the Pacific: A virtual swimming interface. In ACM SIGGRAPH 2004 Emerging technologies (p. 27). ACM.

9. Jain, D., Sra, M., Guo, J., Marques, R., Wu, R., Chiu, J., \& Schmandt, C. (2016, May). Immersive terrestrial scuba diving using virtual reality. In Proceedings of the $2016 \mathrm{CHI}$ Conference Extended Abstracts on Human Factors in Computing Systems (pp. 1563-1569). ACM.

10. Slater, M., \& Wilbur, S. (1997). A framework for immersive virtual environments (FIVE): Speculations on the role of presence in virtual environments. Presence: Teleoperators \& Virtual Environments, 6(6), 603-616.

11. Quan, L., \& Cummings, P. (2003). Characteristics of drowning by different age groups. Injury Prevention, 9(2), 163-168.

12. Witmer, B. G., \& Singer, M. J. (1998). Measuring presence in virtual environments: A presence questionnaire. Presence, 7(3), 225-240.

13. Pandis, N. (2016). The chi-square test. American Journal of Orthodontics and Dentofacial Orthopedics, 150(5), 898899. doi:10.1016/j.ajodo.2016.08.009 